\title{
SEQUENCES NOT CONTAINING AN INFINITE ARITHMETIC PROGRESSION
}

\author{
SAMUEL S. WAGSTAFF, JR. ${ }^{1}$
}

\begin{abstract}
Sequences of integers exist with any possible upper density and lower density which do not contain an infinitely long arithmetic progression.
\end{abstract}

A well-known conjecture in additive number theory states that for every integer $k$ greater than 1 , if an increasing sequence of positive integers does not contain $k$ consecutive terms of an arithmetic progression, then the sequence has density zero. Much work has been done on this problem during the last 40 years, but we have never seen it remarked that the analogous conjecture for infinite arithmetic progressions is false. Namely it is not true that if an increasing sequence of positive integers does not contain an infinitely long arithmetic progression, then the sequence has density zero. (The converse is obviously true.) The following easy theorem shows that the statement is very far from true.

THEOREM. Let $a$ and $b$ be real numbers such that $0 \leqq a \leqq b \leqq 1$. Then there exists an increasing sequence $A$ of positive integers which has lower density $a$ and upper density $b$ and which contains no infinitely long arithmetic progression.

Proof. The sequence $A$ will contain long blocks of consecutive integers separated by large gaps. It is defined by

$$
\begin{aligned}
k_{1}+1, k_{1}+2, \cdots, & k_{1}+l_{1}, k_{1}+l_{1}+k_{2}+1, \cdots, \\
& k_{1}+l_{1}+k_{2}+l_{2}, k_{1}+l_{1}+k_{2}+l_{2}+k_{3}+1, \cdots,
\end{aligned}
$$

where the positive integers $k_{1}, l_{1}, k_{2}, l_{2}, \cdots$ will be defined later. Let $\left\{a_{n}\right\}$ and $\left\{b_{n}\right\}$ be sequences of real numbers satisfying $\lim _{n \rightarrow \infty} a_{n}=a$, $\lim _{n \rightarrow \infty} b_{n}=b$ and

$$
0<a_{n}<b_{n}<1 \text { and } a_{n+1}<b_{n}
$$

for every positive integer $n$ and

$$
b_{n}-a_{n+1}>n^{-1 / 2}
$$

Received by the editors February 29, 1972.

AMS (MOS) subject classifications (1970). Primary $10 \mathrm{~L} 10$.

Key words and phrases. Arithmetic progressions in sequences.

1 The author thanks the Institute for Advanced Study for its hospitality while he did this research.

(c) American Mathematical Society 1973 
for all sufficiently large integers $n$. (Such sequences always exist.) Define the integers $k_{1}, l_{1}, k_{2}, l_{2}, \cdots$ recursively by

$$
\begin{array}{rlrl}
k_{1} & =1, & \\
l_{1} & =1+\left[\frac{b_{1} k_{1}}{1-b_{1}}\right], & \\
k_{n+1} & =1+\left[\left(\frac{1-a_{n+1}}{a_{n+1}}\right) \sum_{i=1}^{n} l_{i}-\sum_{i=1}^{n} k_{i}\right] & (n \geqq 1), \\
l_{n+1} & =1+\left[\left(b_{n+1}\left(\sum_{i=1}^{n} l_{i}+\sum_{i=1}^{n+1} k_{i}\right)-\sum_{i=1}^{n} l_{i}\right) /\left(1-b_{n+1}\right)\right] \quad(n \geqq 6),
\end{array}
$$

where $[x]$ denotes the greatest integer $\leqq x$. Let

$$
S_{n}=\sum_{i=1}^{n-1} l_{i} \text { and } T_{n}=S_{n}+\sum_{i=1}^{n} k_{i}
$$

(The empty sum is zero.) Clearly the partial density of $A$ attains a relative minimum at the end of each gap and a relative maximum at the end of each block. Thus the lower density of $A$ is

$$
\underline{d}(A)=\lim _{n \rightarrow \infty}\left(S_{n} / T_{n}\right)
$$

and the upper density of $A$ is

$$
\bar{d}(A)=\lim _{n \rightarrow \infty}\left(S_{n+1} /\left(T_{n}+l_{n}\right)\right),
$$

if these limits exist. We will show by induction on $n$ that
(a) $k_{n}$ and $l_{n}$ are positive integers,
(b) $a_{n}-1 / T_{n}<S_{n} / T_{n}<a_{n}$, and
(c) $b_{n}<S_{n+1} /\left(T_{n}+l_{n}\right)<b_{n}+1 /\left(T_{n}+l_{n}\right)$.

Statements (a) and (b) are clear for $n=1$. The definition of $l_{1}$ gives $b_{1} /\left(1-b_{1}\right)<l_{1} \leqq 1+b_{1} /\left(1-b_{1}\right)$, whence $b_{1}<l_{1} /\left(1+l_{1}\right) \leqq b_{1}+\left(1-b_{1}\right) /\left(1+l_{1}\right)$, and (c) for $n=1$ follows from $b_{1}>0$. Suppose the three statements are true for $n$; we prove them for $n+1$. From (c) for $n$ and (1), we get

$$
\sum_{i=1}^{n} k_{i}<S_{n+1}\left(\frac{1-a_{n+1}}{a_{n+1}}\right)
$$

so that $k_{n+1}$ is a positive integer. From the definition of $k_{n+1}$ we have

$$
\left(\frac{1-a_{n+1}}{a_{n+1}}\right) S_{n+1}-\sum_{i=1}^{n} k_{i}<k_{n+1} \leqq 1+\left(\frac{1-a_{n+1}}{a_{n+1}}\right) S_{n+1}-\sum_{i=1}^{n} k_{i},
$$

and using $a_{n+1}<1$ we obtain (b) for $n+1$. From $a_{n+1}<b_{n+1}$ and the statement just proved we get $S_{n+1}<b_{n+1} T_{n+1}$, whence $l_{n+1}$ is a positive integer. 
The definition of $l_{n+1}$ gives

Therefore

$$
\frac{b_{n+1} T_{n+1}-S_{n+1}}{1-b_{n+1}}<l_{n+1} \leqq 1+\frac{b_{n+1} T_{n+1}-S_{n+1}}{1-b_{n+1}} .
$$

$$
b_{n+1}<\frac{S_{n+2}}{T_{n+1}+l_{n+1}} \leqq b_{n+1}+\frac{1-b_{n+1}}{T_{n+1}+l_{n+1}}
$$

and (c) for $n+1$ follows from $b_{n+1}>0$. Hence the three statements are true for all positive integers $n$.

Now (a) implies that $\lim _{n \rightarrow \infty} T_{n}=\infty$, so from (b) and (3) we have $\underline{d}(A)=\lim _{n \rightarrow \infty} a_{n}=a$. Similarly (c) and (4) imply that $\bar{d}(A)=b$.

Using (b) for $n$, (c) for $n+1$ and (2) we have for large $n$

$$
n^{-1 / 2}<\frac{S_{n+1}}{T_{n}+l_{n}}-\frac{S_{n+1}}{T_{n+1}}=\frac{S_{n+1} k_{n+1}}{\left(T_{n}+l_{n}\right) T_{n+1}} .
$$

From (a) we have $0<S_{n+1}<T_{n+1}$ and $T_{n}+l_{n}>n$, so $n^{-1 / 2}<k_{n+1} /\left(T_{n}+l_{n}\right)<$ $k_{n+1} / n$. Therefore $k_{n+1}>n^{1 / 2}$ for sufficiently large $n$, so $\lim _{n \rightarrow \infty} k_{n}=\infty$. This implies that $A$ cannot contain an infinitely long arithmetic progression because such a progression could not overleap a gap of length greater than its common difference, and $k_{n}$ is the length of the $n$th gap. This completes the proof.

ADDED IN PROOF. If one only wishes to show that there is a sequence $A$ with density $0 \leqq a<1$ which contains no infinitely long arithmetic progression, one can use the following simple construction suggested by Professor Oscar Rothaus. Let $p_{1}<p_{2}<p_{3}<\cdots$ be primes such that $\prod_{i=1}^{\infty}\left(1-1 / p_{i}\right)=a$, and let $A$ be the set of all natural numbers not divisible by any of the $p_{i}$. Clearly $d(A)=a$. Also $A$ cannot contain an infinite arithmetic progression with common difference $k$ because $k$ would be relatively prime to some $p_{i}$, and then one term of the arithmetic progression would be a multiple of this $p_{i}$.

School of Mathematics, Institute for Advanced Study, Princeton, New JERSEY 08540 61801

Current address: Department of Mathematics, University of Illinois, Urbana, Illinois 